\title{
Inducible Nitric Oxide Synthase Suppresses the Development of Allograft Arteriosclerosis
}

\author{
Larry L. Shears II," Nobuyoshi Kawaharada, ${ }^{*}$ Edith Tzeng, ${ }^{\star}$ Timothy R. Billiar, ${ }^{\star}$ Simon C. Watkins, ${ }^{\ddagger}$ Imre Kovesdi, \\ Alena Lizonova, $\$$ and Si M. Pham* \\ $*$ Departments of Surgery and ${ }^{\ddagger}$ Center for Biologic Imaging, University of Pittsburgh, Pittsburgh, Pennsylvania 15261; and ${ }^{\S}$ GenVec \\ Corporation, Rockville, Maryland 20852
}

\begin{abstract}
In cardiac transplantation, chronic rejection takes the form of an occlusive vasculopathy. The mechanism underlying this disorder remains unclear. The purpose of this study was to investigate the role nitric oxide (NO) may play in the development of allograft arteriosclerosis. Rat aortic allografts from ACI donors to Wistar Furth recipients with a strong genetic disparity in both major and minor histocompatibility antigens were used for transplantation. Allografts collected at $28 \mathrm{~d}$ were found to have significant increases in both inducible NO synthase (iNOS) mRNA and protein as well as in intimal thickness when compared with isografts. Inhibiting NO production with an iNOS inhibitor increased the intimal thickening by $57.2 \%$, indicating that NO suppresses the development of allograft arteriosclerosis. Next, we evaluated the effect of cyclosporine (CsA) on iNOS expression and allograft arteriosclerosis. CsA (10 mg/kg/d) suppressed the expression of iNOS in response to ballooninduced aortic injury. Similarly, CsA inhibited iNOS expression in the aortic allografts, associated with a $65 \%$ increase in intimal thickening. Finally, we investigated the effect of adenoviral-mediated iNOS gene transfer on allograft arteriosclerosis. Transduction with iNOS using an adenoviral vector suppressed completely the development of allograft arteriosclerosis in both untreated recipients and recipients treated with CsA. These results suggest that the early immune-mediated upregulation in iNOS expression partially protects aortic allografts from the development of allograft arteriosclerosis, and that iNOS gene transfer strategies may prove useful in preventing the development of this otherwise untreatable disease process. (J. Clin. Invest. 1997. 100: 2035-2042.) Key words: gene therapy • transplantation • intimal hyperplasia $\bullet$ chronic rejection $\cdot$ cyclosporine
\end{abstract}

\section{Introduction}

Acute irreversible rejection is now a rare cause of clinical organ allograft failure. Instead, chronic rejection, in its various

\footnotetext{
Address correspondence to Si M. Pham, M.D., Suite C700, Presbyterian University Hospital, 200 Lothrop Street, Pittsburgh, PA 15213. Phone: 412-648-9758; FAX: 412-648-1029; E-mail: pham@pittsurg. nb.upmc.edu

Received for publication 28 May 1997 and accepted in revised form 13 August 1997.
}

J. Clin. Invest.

(C) The American Society for Clinical Investigation, Inc. 0021-9738/97/10/2035/08 \$2.00

Volume 100, Number 8, October 1997, 2035-2042

http://www.jci.org organ-specific forms has proven to be the major obstacle to long-term allograft function. In cardiac transplant recipients, the development of accelerated coronary arteriosclerosis has become the principle cause of late death and allograft dysfunction (1-3). This disease can be detected by coronary angiography in $6-18 \%$ of cardiac recipients at $1 \mathrm{yr}$ and in as many as $50 \%$ at $5 \mathrm{yr}(4,5)$. Based on descriptive studies, the hallmark manifestation of this pathologic disease process is a diffuse, progressive narrowing of the coronary vessels due to intimal hyperplasia $(1,6,7)$. Similar to restenosis after balloon angioplasty, allograft arteriosclerosis involves the abnormal proliferation and migration of vascular smooth muscle cells (VSMC $)^{1}$ from their normal position in the tunica media to the progressively enlarging intimal layer (8). While our current understanding of this disease process is incomplete, leading hypotheses suggest that incompletely suppressed cellular and humoral immune responses initiate cytokine and growth factor cascades that elicit an immune-mediated endothelial injury (9-11). These alloimmune responses are directed towards disparate major histocompatibility antigens expressed on the endothelial surfaces of the allograft (12). The ensuing endothelial dysfunction and injury result in the exposure of the underlying VSMC to mitogenic growth factors (13). This is followed by the phenotypic conversion of the VSMC from a normally contractile nonproliferating phenotype to the pathologic secretory proliferating phenotype observed in both restenosis and allograft arteriosclerosis $(14,15)$.

Recent studies have determined that the cytokine-inducible isoform of nitric oxide (NO) synthase (iNOS or NOS-2) is upregulated during both the acute and chronic stages of vascular rejection (16-18). Early on, iNOS is expressed predominately in the infiltrating inflammatory cells that invade the subendothelial and periadventitial layers, and is considered to be a marker of both macrophage activation and acute cellular rejection (19). In the later stages of chronic cardiac rejection, iNOS expression can be detected in the VSMC of the medial and the neointimal layers (20). The consequences of this intramural upregulation of iNOS are unknown. NO is known perhaps most notably for its physiologic regulation of vasomotor tone and its ability to inhibit platelet aggregation $(21,22)$. In addition to these more commonly known functions, $\mathrm{NO}$ also suppresses $\mathrm{T}$ cell proliferation and inhibits leukocyte chemotaxis, lending support to an immunomodulatory role for $\mathrm{NO}$ during allograft rejection (23-25). Additionally, the ability of NO to inhibit VSMC proliferation has been exploited in gene transfer strategies as a means of inhibiting neointimal hyperplasia after balloon angioplasty, illustrating that NO may pro-

1. Abbreviations used in this paper: CsA, cyclosporine A; iNOS, inducible NO synthase; L-NIL, L- $N^{6}-(1$-iminoethyl)-lysine; NO, nitric oxide; RT-PCR, reverse transcriptase-PCR; VSMC, vascular smooth muscle cells. 
vide important antiatherogenic properties (26-28). It is interesting to note that cyclosporine A (CsA), which is commonly used to suppress allograft rejection, has been shown to accelerate allograft arteriosclerosis in some models, and is known to inhibit iNOS expression in vitro (29-31). Again, this raises the possibility that intramural iNOS expression directly suppresses intimal hyperplasia. NO has also been implicated in the tissue injury associated with several chronic inflammatory and autoimmune diseases, such as diabetes mellitus and rheumatoid arthritis (32). Thus, the question that arises is whether the potentially cytotoxic actions of $\mathrm{NO}$ or its reaction products perpetuate the pathogenesis of allograft arteriosclerosis or whether the antiatherogenic properties of NO suppress the development of neointimal hyperplasia and impede the development of chronic rejection in cardiac allografts.

Experiments were undertaken here to determine the role iNOS plays in allograft arteriosclerosis in an aortic allograft model of chronic rejection. Using a partially selective iNOS inhibitor, we determine that iNOS expression in aortic allografts suppresses the development of allograft arteriosclerosis. Additionally, we report that CsA inhibits iNOS expression not only in vitro, but also in vivo, and that this inhibition contributes to the CsA-mediated acceleration of allograft arteriosclerosis. Finally, we demonstrate the efficacy of iNOS gene transfer to inhibit allograft arteriosclerosis.

\section{Methods}

Animals. Male Wistar Furth (WF, RT1A $\left.{ }^{\mathrm{u}}\right)$ and ACI $\left(\mathrm{RT}^{\mathrm{A}} \mathrm{A}^{\mathrm{a}}\right)$ rats were purchased from Harlan Sprague Dawley Inc. (Indianapolis, IN). Rats 1-3 mo of age weighing 200-250 g were used as donors and recipients. All animals received humane care in compliance with the Principles of Laboratory Animal Care and the Guide for the Care and Use of Laboratory Animals, prepared by the Institute of Laboratory Animal Resources and published by the National Institutes of Health (NO86-23, revised 1985).

Aortic transplantations. The descending thoracic aorta was excised from donor rats after cauterization of the intercostal arteries. The aortic segment was perfused with saline and used for transplantation. Recipient animals were intubated and anesthetized with methoxyflurane. The aortic graft $(3 \mathrm{~cm}$ in length) was transplanted into a heterotopic position below the renal arteries and above the aortic bifurcation, as described by Mennander and colleagues (33). All anastomoses were performed using continuous 9-0 nylon suture in an end-to-end fashion. Grafts were removed $28 \mathrm{~d}$ after transplantation and divided into three equal $10-\mathrm{mm}$ segments. The cranial and caudal segments were immediately frozen in liquid nitrogen and stored at $-80^{\circ} \mathrm{C}$ for later RNA extraction and reverse transcriptase-PCR (RTPCR) analyses. The center segment was fixed with $2 \%$ paraformaldehyde, dehydrated with $30 \%$ sucrose, and frozen in OCT medium (Tissue Tek; Miles Laboratories, Inc., Elkhart, IN) for later immunohistochemical and morphometric evaluations.

NOS-inhibitor experiments. The ACI-to-WF strain combination was used for all allogeneic transplantations. This strain combination involves a complete disparity in both major and minor histocompatibility antigens. ACI-to-ACI isografts served as our syngeneic controls. To determine what effect chronic iNOS expression has on the progression of allograft arteriosclerosis, one group of animals receiving allogeneic grafts was treated with a continuous infusion of L- $\mathrm{N}^{6}$ (1-iminoethyl)-lysine (L-NIL; Cayman Chemical Co., Inc., Ann Arbor, MI), an inhibitor that exhibits 33-50-fold more selectivity for iNOS than endothelial NOS (34). This drug was delivered at a rate of $20 \mu \mathrm{mol} / \mathrm{kg} / \mathrm{d}$ for $28 \mathrm{~d}$ using a miniosmotic pump (Alzet; ALZA Corp., Palo Alto, CA) implanted so that the catheter from the pump was positioned in the jugular vein at the time of transplantation. The pump itself was placed in a subcutaneous pocket on the dorsum of the animal. Control allografted animals received a continuous infusion of saline from the miniosmotic pumps placed in an identical manner. The aortic grafts were excised after $28 \mathrm{~d}$ as described above for morphometric analyses.

To determine whether CsA alters iNOS expression after transplantation, allogeneic and syngeneic aortic transplants were performed as described above. Animals were randomized to receive either no treatment or CsA $(10 \mathrm{mg} / \mathrm{kg} / \mathrm{d})$ administered subcutaneously throughout the duration of the experiments. Grafts were excised after $4 \mathrm{wk}$ as previously described. The cranial and caudal segments were used for RT-PCR analyses, and the center segment was used for histomorphometric examinations.

CsA experiments. CsA is known to inhibit iNOS in vitro. To determine whether this also occurs in vivo, a 2 French Fogarty catheter was introduced into the thoracic aorta via the left carotid artery of male Sprague-Dawley rats. The catheter was inflated to 2 atmospheres and then drawn through the thoracic aorta twice. This injury model induces iNOS expression in the tunica media for $\sim 2 \mathrm{wk}(35)$. Animals were randomized to receive either saline or CsA $(10 \mathrm{mg} / \mathrm{kg} / \mathrm{d})$ administered subcutaneously. Aortas were isolated $48 \mathrm{~h}$ later and frozen immediately in liquid nitrogen for later RNA extraction and RT-PCR analysis.

Preparation of adenoviral vectors. The human iNOS cDNA was cloned previously from cytokine-stimulated human hepatocytes (33). An E1- and E3-deleted vector carrying the human iNOS cDNA was designed and constructed. Briefly, an adenovirus transfer plasmid with the cytomegalovirus promoter and an artificial splice sequence was designed to drive the transcription of the human iNOS cDNA. To generate infectious virus, the plasmid was cotransfected into 293 cells (CRL. 1573; American Type Culture Collection, Rockville, MD) with the large C1aI fragment of AdlacZ DNA (36). Intracellular recombination of the plasmid with the adenovirus DNA fragment generated a full-length recombinant adenoviral genome (37). Recombinant AdiNOS virus was double plaque-purified and screened for nitrite $\left(\mathrm{NO}_{2}^{-}\right)$accumulation using the Griess reaction (38). Viral stocks were purified by triple banding on a cesium chloride gradient. Concentrations of AdiNOS and the control adenovirus AdlacZ were determined by plaque assay. The titer of AdiNOS was $10^{9}$ plaqueforming units $(\mathrm{pfu}) / \mathrm{ml}$, and AdlacZ was $10^{10} \mathrm{pfu} / \mathrm{ml}$. Both vectors were diluted to a concentration of $10^{7} \mathrm{pfu} / \mathrm{ml}$ for aortic transfections.

iNOS gene transfer strategy. To determine whether overexpression of iNOS influenced the development of allograft arteriosclerosis, we developed an iNOS gene transfer strategy to increase the local production of NO in the aortic allografts. The donor thoracic aortas were excised and then immersed in $2 \mathrm{ml}$ of a transfection medium consisting of Optimem I (GIBCO BRL, Gaithersburg, MD) and containing an adenoviral vector that carried either the human iNOS cDNA (AdiNOS) or the cDNA encoding the $\beta$-galactosidase gene (AdlacZ). Viral titers were $10^{7} \mathrm{pfu} / \mathrm{ml}$ for each vector, and the transfection period was $60 \mathrm{~min}$ at $27^{\circ} \mathrm{C}$. To prove that this gene transfer strategy increased NO production in the aortic grafts, AdlacZ- and AdiNOS-transduced grafts were placed into an arterial organ culture system and incubated at $37^{\circ} \mathrm{C}$ in DME supplemented with $20 \%$ FCS for $24 \mathrm{~h} \mathrm{(28).} \mathrm{NO}_{2}{ }^{-}$accumulation in the supernatant was determined using the Griess reaction.

To determine whether the AdiNOS gene transfer altered the development of allograft arteriosclerosis, the transduced aortic grafts were transplanted as described above. Allografted aortas were isolated after $4 \mathrm{wk}$ for morphometric analysis. To determine whether iNOS gene transfer reversed the increased allograft arteriosclerosis observed in CsA-treated grafts, a second group of rats receiving AdiNOS-treated grafts was injected with CsA $(10 \mathrm{mg} / \mathrm{kg} / \mathrm{d})$ for $28 \mathrm{~d}$.

Histology and morphometry. After immersion-fixation with $2 \%$ paraformaldehyde and tissue cryoprotection with $2.3 \mathrm{M}$ sucrose, $5-\mu \mathrm{m}$ sections were cut from three random sites in the middle third of each graft. Sections were stained using Mayer's hematoxylin and eosin and Verhoff/van Geison's elastin staining techniques. Images were col- 

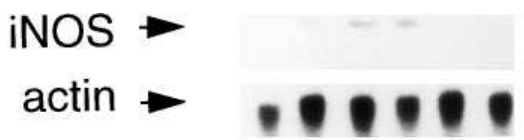

4 Isografts

\section{iNOS

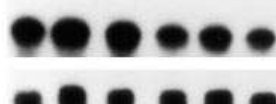 \\ actin

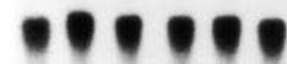 \\ Allografts}

Figure 1. iNOS mRNA expression in aortic allografts. Syngeneic $(n=6)$ and allogeneic $(n=6)$ transplanted aortas were collected $28 \mathrm{~d}$ after transplantation. The cranial and caudal thirds of the aortic grafts were frozen immediately in liquid nitrogen for RT-PCR analysis. Semiquantitative RT-PCR using ${ }^{32} \mathrm{P}$-labeled iNOS primers was performed on RNA extracted from the aortic segments. Actin served as a control to ensure adequate cDNA was obtained from all specimens. PCR products were separated on a $5 \%$ polyacrylamide gel and exposed on an autoradiograph. Low levels of iNOS mRNA were detected in three of the six syngeneic grafts. All allogeneic grafts had high levels of iNOS mRNA expression.

lected directly using a microscope with a $\times 10$ objective (Nikon Inc., Melville, NY), a high sensitivity, integrating three-chip color camera (Sony Corp., Park Ridge, NJ) $(700 \times 600$ pixels), and a frame grabber board (Coreco Inc., St.-Laurent, Quebec, Canada). Measurements of the intimal and medial layer thicknesses were taken at 15 separate sites and recorded as the mean intimal and medial layer thicknesses, using image-analysis software (Optimas Corp., Seattle, WA).

Immunohistochemistry. Immunohistochemistry was performed on cryostat sections ( $5 \mu \mathrm{m}$ thick) prepared as above using an immunofluorescent technique. Sections were washed three times with PBS containing $0.5 \%$ BSA and $0.15 \%$ glycine, $\mathrm{pH} 7.4$ (buffer A). This was followed by a 60 -min incubation with purified goat $\operatorname{IgG}(50 \mathrm{mg} / \mathrm{ml})$ at $25^{\circ} \mathrm{C}$ and three additional washes with buffer $\mathrm{A}$ in order to ensure minimal nonspecific reaction to the primary and secondary antibodies. The sections were then incubated for $60 \mathrm{~min}$ with the primary an- tibody, iNOS (1:300 dilution) (Transduction Laboratories, Lexington, $\mathrm{KY}$ ). This was followed by three additional washes with buffer A and a 60-min incubation period with the secondary antibody (Streptavidin Cy3, dilution 1:3,000; Amersham Corp., Arlington Heights, IL). The sections were then washed again with buffer A three times, mounted in Gelvatol (Monsanto Corp., St. Louis, MO), and coverslipped for fluorescence microscopy.

$R T-P C R$. Total cellular RNA was extracted from the isolated aortic segments with RNAzol as previously described (39). Total RNA $(2.5 \mu \mathrm{g})$ was subjected to reverse transcription with $20 \mathrm{U} / \mathrm{ml}$ Moloney leukemia virus reverse transcriptase (GIBCO BRL) for 60 min at $37^{\circ} \mathrm{C}$. PCR was performed on a thermocycler (Perkin-Elmer Corp., Norwalk, CT), using 30 cycles in a $50-\mu l$ reaction volume including 1.25 U Taq DNA polymerase (Perkin-Elmer), $10 \mathrm{nM}$ of each primer, and $1.0 \mu \mathrm{l} \mathrm{cDNA}$ template. Primers for iNOS were $3^{\prime}$-TTGGGTCTTGTTAGCCTA and 5'-TGTGCAGTCCCAGTGAGGAACGTC, defining a fragment of $162 \mathrm{bp}$. Amplification of $\alpha$-actin RNA served as a control to ensure that adequate PCR products were obtained from each specimen. The $5^{\prime}$ primer was labeled with phosphorus-32 using $\mathrm{T}_{4}$ polynucleotide kinase (USB Biologicals, Cleveland, $\mathrm{OH})$ and $\left[\gamma^{32} \mathrm{P}\right] \mathrm{dATP}$. The PCR products were separated by electrophoresis on a $5 \%$ polyacrylamide gel, and visualized by autoradiography and via a PhosphorImager (Phosphor; Molecular Dynamics, Sunnyvale, CA).

Statistics. Mean thicknesses of the intimal and medial layers and intimal/medial ratios for groups of experimental and control vessels were compared using ANOVA. If the ANOVA was found to be statistically significant $(P<0.05)$, pairwise comparisons were performed using Fisher's exact test. Six animals were analyzed in each group. Data are presented as mean \pm SEM.

\section{Results}

Effects of the alloimmune response on iNOS expression. The rat aortic allograft model was used to determine the pattern of iNOS expression during the alloimmune response. Syngeneic and allogeneic grafts were harvested $28 \mathrm{~d}$ after transplantation, and iNOS mRNA was measured by RT-PCR. As shown in Fig. 1, low-level iNOS mRNA expression was detected in three of six aortic isografts. In contrast, iNOS mRNA expres-
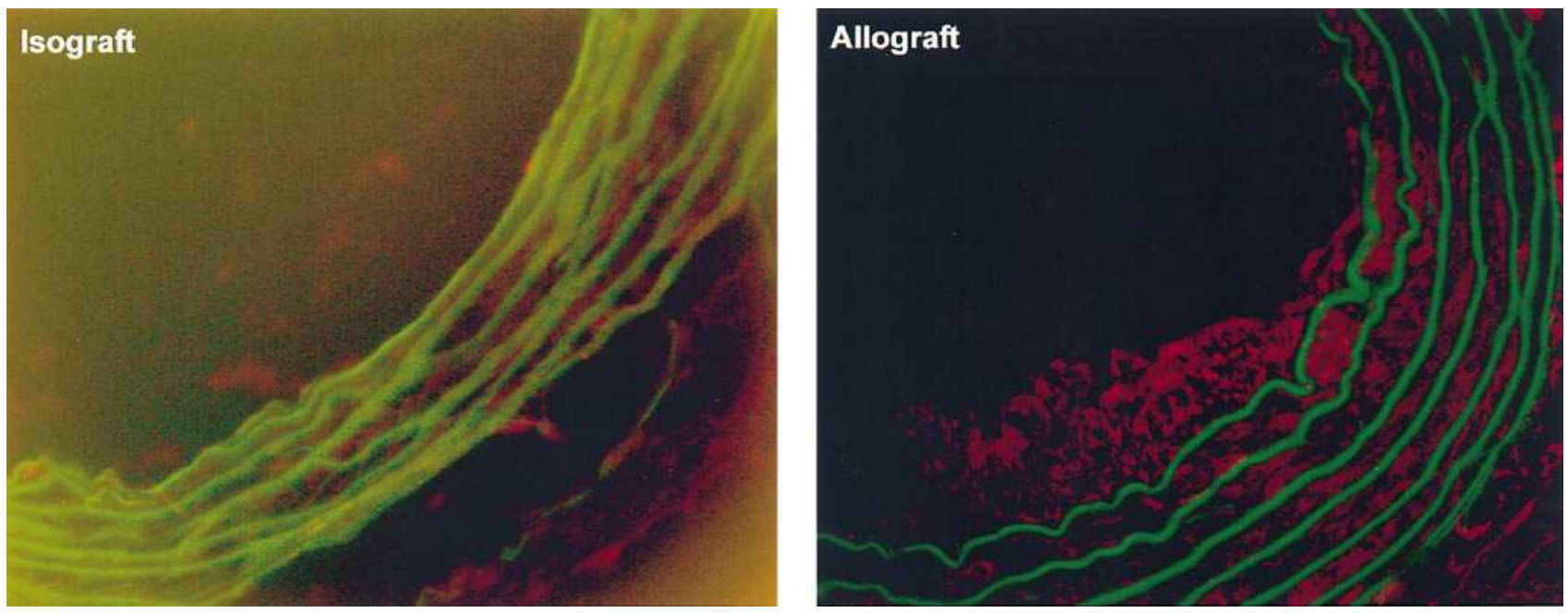

Figure 2. Immunohistochemistry for iNOS protein in aortic allografts. Syngeneic and allogeneic aortic grafts were collected $28 \mathrm{~d}$ after heterotopic transplantation. The center portion of each graft was immersion-fixed in $2 \%$ paraformaldehyde frozen in OCT. Sections from these segments were probed for iNOS protein expression using an anti-iNOS antibody (dilution 1:300) for 60 min. Protein binding by the anti-iNOS antibody was visualized using fluorescently tagged secondary antibodies (dilution 1:3,000) to show iNOS protein (orange) in isografted and allografted aortas. 
A

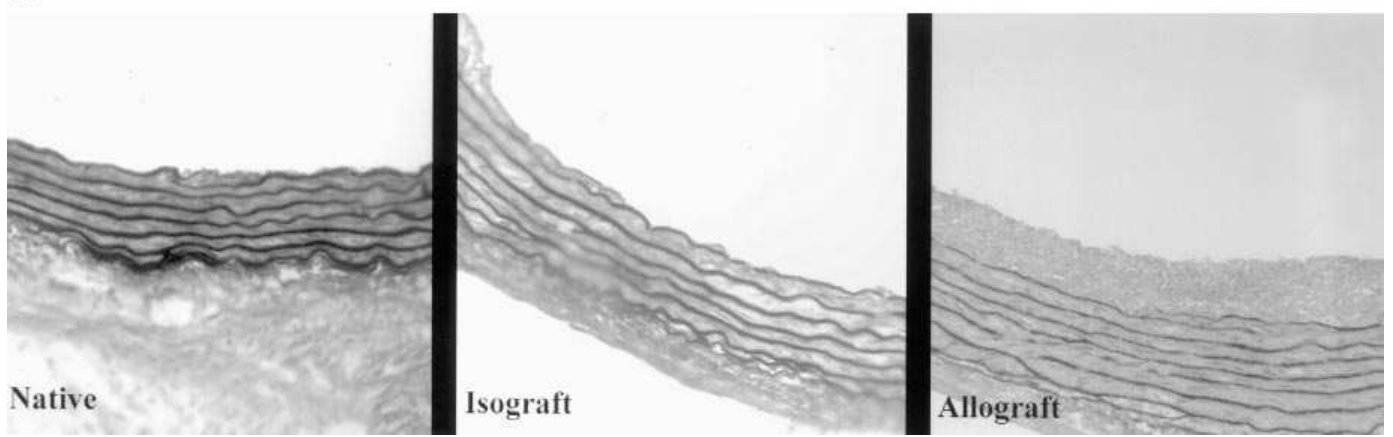

B

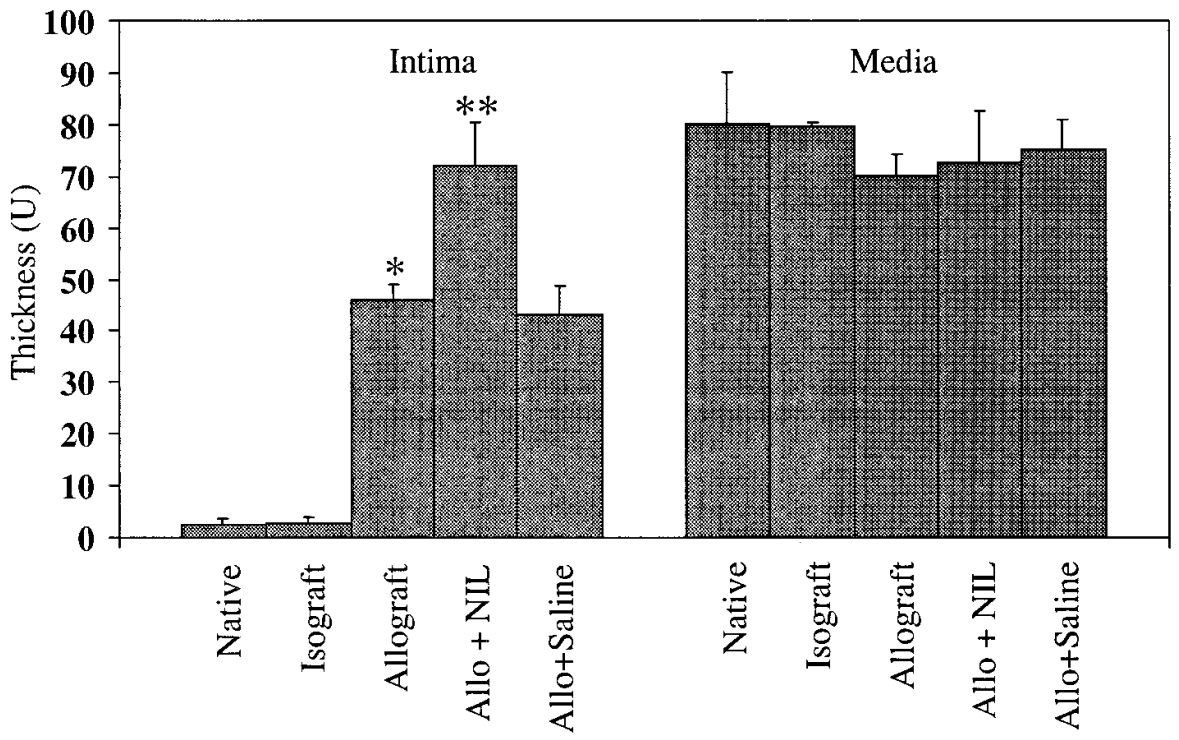

Figure 3. Effects of induced nitric oxide on allograft arteriosclerosis. Syngeneic $(n=6)$ and allogeneic $(n=6)$ untreated aortic transplants were collected after $28 \mathrm{~d}$. Some rats receiving an aortic allograft received a continuous infusion of either saline or the selective iNOS inhibitor L-NIL for $28 \mathrm{~d}$ after transplant via a miniosmotic pump. Grafts were collected after 4 wk. The center third of the grafts was perfused with $2 \%$ paraformaldehyde and frozen in Gelvatol. Sections were stained with hematoxylin and eosin and Verhoff/van Geison stains, and intimal and medial thicknesses were determined using a semiautomated computer analysis program. $(A)$ Histology comparing isografts and allografts to native aortas; $(B)$ intimal and medial thicknesses of allografts treated with a continuous infusion of L-NIL or saline. $* P<0.05$ compared to isograft. $* * P<0.05$ compared to allograft. sion was upregulated significantly in all six of the allografts, demonstrating that the alloimmune response induces vascular iNOS expression.

Immunohistochemical studies confirmed the increase in iNOS protein in allogeneic grafts when compared with the syngeneic controls. As shown in Fig. 2, iNOS protein was detected at very low levels in the aortic isografts, as expected, based upon the RT-PCR data. Significantly more iNOS protein was detected in the aortic allografts, with most of the iNOS protein detected in the hyperplastic intimal and medial layers of the arterial wall.

Effects of iNOS expression on allograft arteriosclerosis. Neointimal thickness was determined in all grafts by measuring the distance from the endothelium to the internal elastic lamina. As shown in Fig. $3 A$, no significant increase in intimal thickness was noted in the isografts when compared with the nontransplanted native aortas. In contrast, allografts exhibited a 15.9-fold increase in intimal thickness at $4 \mathrm{wk}$, demonstrating that the alloimmune response initiates intimal hyperplasia.

To determine the role allograft iNOS expression plays in the progression of allograft arteriosclerosis, we measured the intimal thickening in allografts receiving a continuous infusion of either L-NIL (iNOS inhibitor) or saline via implanted miniosmotic pumps. As shown in Fig. $3 \mathrm{~B}$, sustained iNOS inhibition increased significantly the intimal thickening over that ob- served in the saline controls, suggesting a role for induced NO in impeding the development of allograft arteriosclerosis.

Effects of CsA on iNOS expression in vivo. CsA inhibits NO production in cytokine-stimulated VSMC in vitro by inhibiting iNOS transcription (19). To demonstrate that CsA inhibits iNOS expression in vivo at therapeutically relevant doses, we

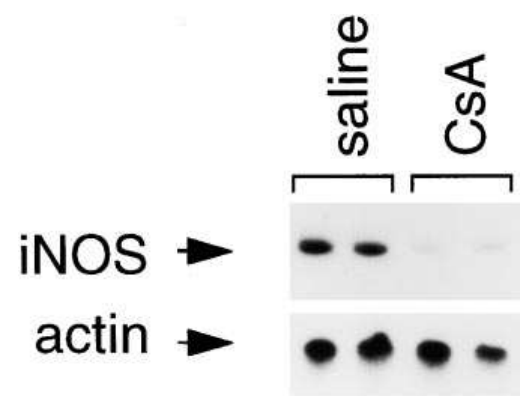

Figure 4. Effect of CsA on iNOS expression and arteriosclerosis. A 2 French Fogarty catheter was inserted into the carotid artery, advanced into the thoracic aorta, and inflated to 2 atmospheres. The catheter was then drawn through the aorta two times. Ani-

mals were randomized to receive either CsA $(10 \mathrm{mg} / \mathrm{kg} / \mathrm{d})$ or saline injections daily for $2 \mathrm{~d}$, after which the animals were killed, and the thoracic aortas were harvested. Semiquantitative RT-PCR using ${ }^{32} \mathrm{P}-$ labeled iNOS primers was performed on RNA isolated from the aortas. Detection of $\alpha$-actin served as a control for cDNA synthesis. 
A

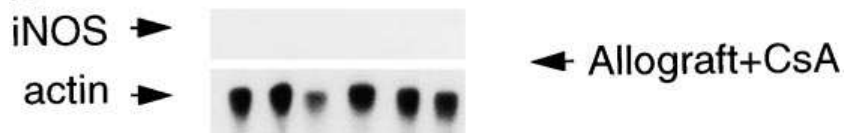

B

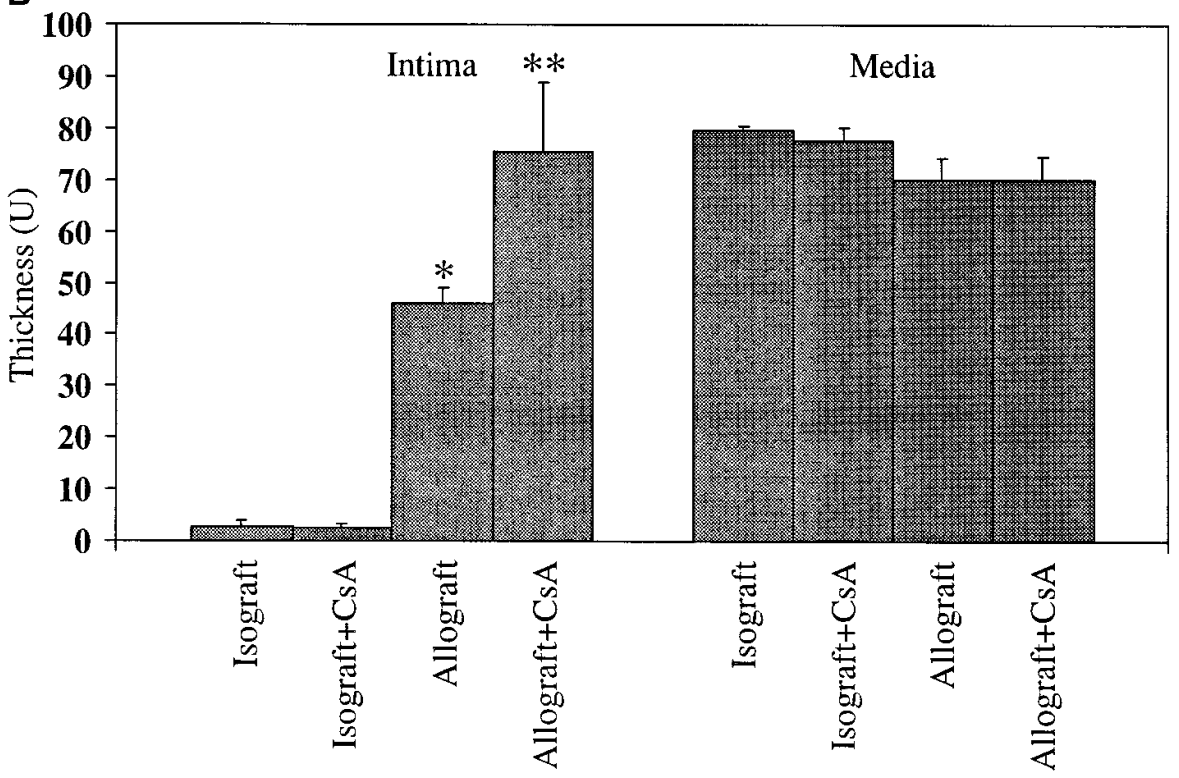

Figure 5. Effect of CsA on iNOS expression and allograft arteriosclerosis. Syngeneic $(n=6)$ and allogeneic $(n=6)$ aortic transplants were harvested after $28 \mathrm{~d}$. The cranial and caudal thirds of each graft were frozen immediately in liquid nitrogen for RNA extraction and RT-PCR analyses. The center segment was fixed with $2 \%$ paraformaldehyde for histomorphometric analysis. ( $A$ ) Semiquantitative RT-PCR using ${ }^{32} \mathrm{P}$-labeled iNOS primers was used to detect iNOS expression in the aortic allografts. No iNOS mRNA was detectable in the CsA-treated allografts. (B) The aortic allografts were stained with hematoxylin and eosin and Verhoff/van Geison stains, and the intimal and medial thicknesses were calculated using a semiautomated computer analysis program. Intimal and medial thicknesses in syngeneic grafts treated with CsA were not significantly thicker than untreated syngeneic grafts. Intimal thicknesses in allogeneic grafts treated with CsA were 1.6-fold thicker than those observed in untreated allogeneic grafts. $* P<0.05$ compared to isograft $* * P<0.05$ compared to allograft. treated rats that had undergone aortic balloon injury with CsA $(10 \mathrm{mg} / \mathrm{kg} / \mathrm{d})$. Prior studies have shown that iNOS can be detected in similarly injured arteries for up to $14 \mathrm{~d}$ (35). As illustrated in Fig. 4, balloon injury induces iNOS expression in the injured aortic segments. Animals receiving CsA after balloon injury were found to have significantly lower levels of iNOS expression than the saline-treated animals that had also been injured. This evidence indicates that CsA can inhibit iNOS expression in vivo at therapeutically relevant dosages.

We next tested the effects of CsA on iNOS expression in the aortic allograft model. As illustrated in Fig. 1, the alloimmune response induces iNOS expression in the aortic allografts. Allografts from CsA (10 mg/kg/d)-treated rats, however, had no detectable iNOS mRNA expression (Fig. $5 \mathrm{~A}$ ). We next examined the effects of CsA on allograft arteriosclerosis. Fig. $5 B$ shows that the intimal thickness in isografts treated with CsA does not increase over that observed in the untreated isografts. However, the intimal thickness in the CsA-treated allograft group was $65 \%$ greater than that observed in the untreated allografts. This finding indicates that the increase in intimal hyperplasia observed with CsA treatment does not occur in the absence of an alloimmune response, and that the CsA-mediated acceleration in allograft arteriosclerosis correlates with a suppression in iNOS expression.

Effect of iNOS gene transfer on allograft arteriosclerosis. To determine whether iNOS overexpression would modify the allograft intimal hyperplasia in the presence or absence of CsA, allografts were transduced using an adenovirus vector containing either the human iNOS cDNA or the cDNA for $\beta$-galactosidase. To establish that iNOS activity was increased in the AdiNOS-transduced vessels, some grafts were placed in an organ culture system, and $\mathrm{NO}_{2}^{-}$release was measured over a 24-h period. As shown in Fig. $6 \mathrm{~A}$, only the AdiNOS-transduced vessels exhibited a significant increase in $\mathrm{NO}_{2}{ }^{-}$release. As shown in Fig. 6, $B$ and $C$, transduction of allografts with the AdiNOS vector inhibited significantly the development of allograft arteriosclerosis measured at $4 \mathrm{wk}$, while AdlacZtreated allografts exhibited no difference from nontransduced allografts. Additionally, AdiNOS-transduced grafts placed in animals treated with CsA also failed to develop significant intimal thickening, thus abrogating completely the accelerated development of allograft arteriosclerosis noted in allografts from the CsA-treated controls (Fig. $6 \mathrm{D}$ ). Unlike endogenously expressed iNOS, the iNOS transgene should not be subject to transcriptional suppression (Fig. 7), since the promoter of the gene was replaced with a cytomegalovirus promoter. As shown in Fig. 7, iNOS mRNA levels were not reduced in the VSMC of rats transduced with iNOS and given $\operatorname{CsA}\left(10^{-4} \mathrm{M}\right)$.

\section{Discussion}

In this study, we demonstrate that the alloimmune response induces the sustained intramural expression of iNOS in rejecting aortic allografts. Similar to the findings in cardiac allografts reported by Russell et al. (20), we found that iNOS expression is localized primarily to the VSMC in the medial and neointimal layers. With the established capacity of NO to limit intimal hyperplasia in vascular injury models (40-42), we hypothesized that the intramural upregulation of iNOS in the aortic allografts would suppress the development of allograft arteriosclerosis. This hypothesis was supported by our experiments showing that continuous inhibition of iNOS activity using a partially selective iNOS inhibitor increased the development 

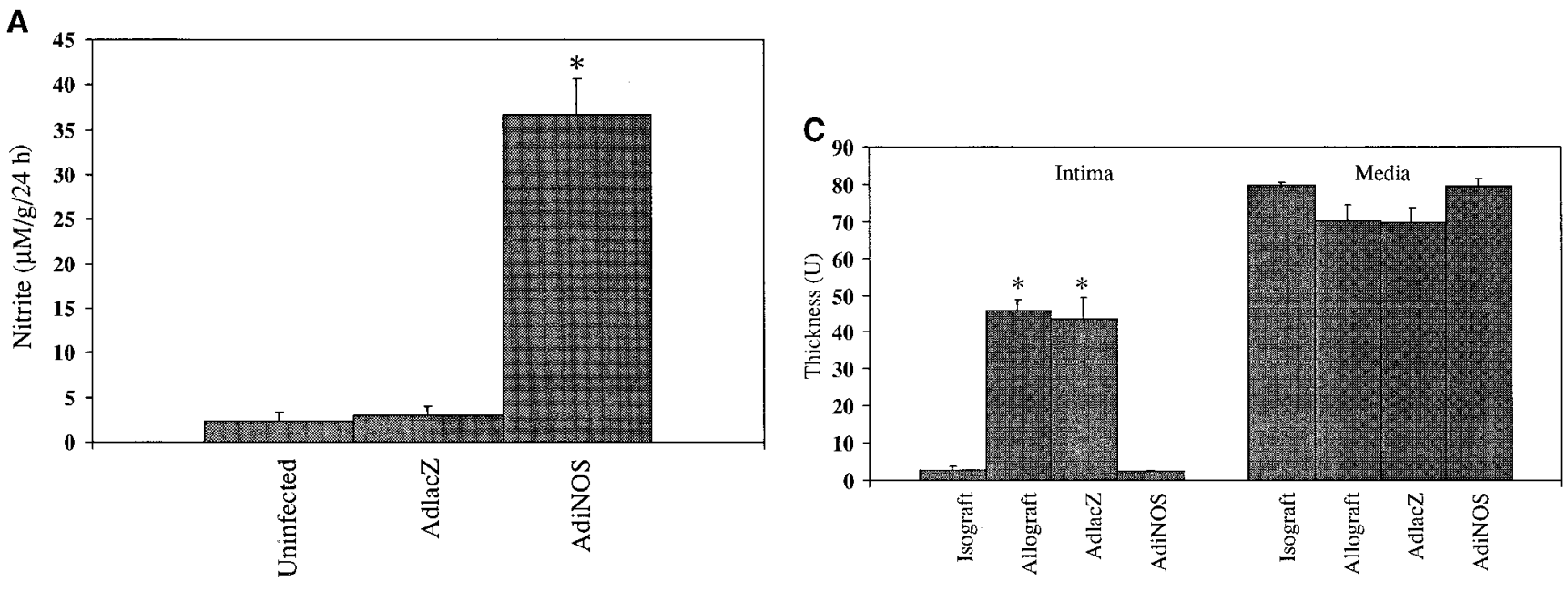

B

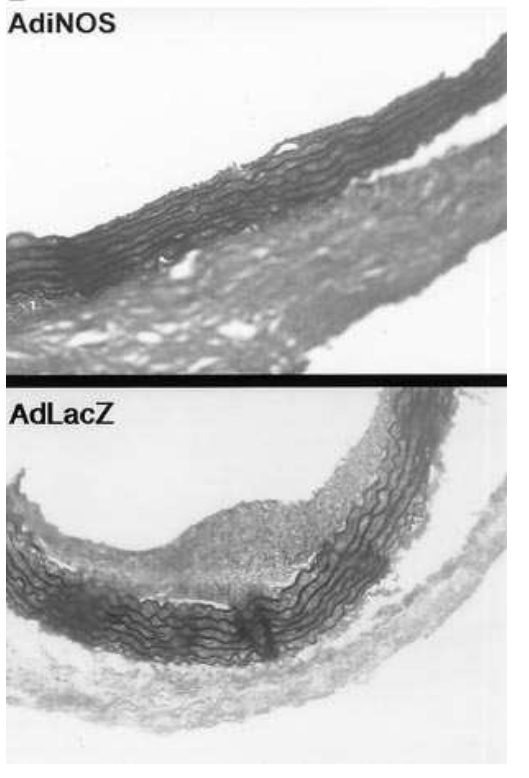

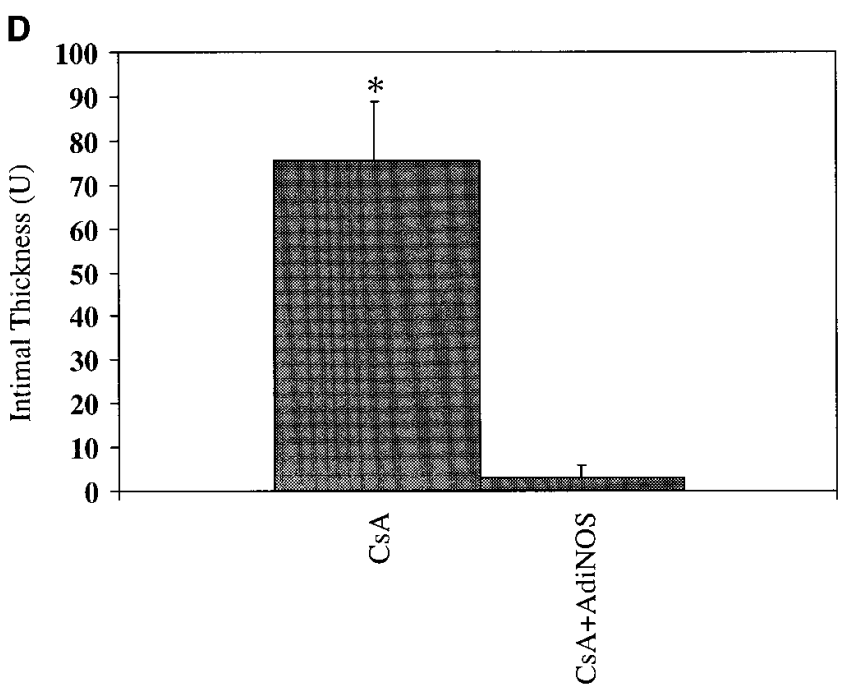

Figure 6. (A) Aortic segments were transduced with either AdiNOS $\left(10^{6} \mathrm{pfu} / \mathrm{ml}\right)$ or AdlacZ $\left(10^{6} \mathrm{pfu} / \mathrm{ml}\right)$ for $1 \mathrm{~h}$. The vessels were then placed in an organ culture system consisting of DME supplemented with $10 \%$ FCS. Media were collected over a 24 -h time period for measurement of $\mathrm{NO}_{2}{ }^{-}$accumulation. Vessels transduced with AdlacZ had no significant change in $\mathrm{NO}_{2}{ }^{-}$accumulation over nontransduced aortic segments, while AdiNOS-treated vessels had significant increases in $\mathrm{NO}_{2}{ }^{-}$accumulation. $* P<0.05$ compared to uninfected grafts. ( $B$ and $C$ ) ACI

aortic grafts transduced with either the AdlacZ $(n=6)$ or the AdiNOS $(n=6)$ vectors were transplanted into WF recipients. Aortas were collected $28 \mathrm{~d}$ later for histomorphometric analyses. Allograft arteriosclerosis in grafts treated with AdiNOS was nearly completely suppressed, while allografts treated with AdlacZ were not significantly different than untransduced allogeneic controls. $* P<0.05$ compared to isograft. $(D)$ AdiNOS transfection also inhibited the accelerated development of allograft arteriosclerosis in CsA-treated allografts. $* P<0.05$ compared to CSA + AdiNOS.

of allograft arteriosclerosis. The clinical relevance of this relationship was demonstrated by our experiments showing that CsA inhibits iNOS expression in allografts in association with a dramatic increase in intimal hyperplasia. The importance of iNOS in this response was confirmed by experiments showing that iNOS gene transfer effectively inhibits intimal hyperplasia in allografts both in the presence and absence of CsA. Therefore, we conclude that iNOS expressed early after transplantation suppresses the short-term development of allograft arteriosclerosis, and that overexpression of iNOS effectively blocks allograft arteriosclerosis for the first $4 \mathrm{wk}$.

NO has multiple, well-described actions in the circulation. In healthy blood vessels, NO produced by the constitutive endothelial NOS (eNOS or NOS-3) found in endothelial cells (43) regulates basal vascular tone (21), prevents platelet activation (22), and limits leukocyte adhesion to the endothelium
(23-25). Most types of vascular cells, including VSMC, can express iNOS in response to vascular injury or inflammation. This induction is mediated by cytokines such as IL- $1 \beta$, TNF- $\alpha$, and IFN- $\gamma$ (44). Factors derived from blood (thrombin, plasmin) and from vascular cells (PDGF, TGF- $\beta$, IGF, EGF, and bFGF) also regulate iNOS expression in VSMC at sites of injury, and may contribute to the local control of blood flow in the absence of a normally protective endothelium. NO can also inhibit VSMC proliferation and migration. Thus, iNOS expression after endothelial denuding injuries may serve to regulate the smooth muscle response and vascular remodeling after vascular injury. Indeed, methods to increase NO availability have been shown to inhibit restenosis after arterial injury.

iNOS has been shown to be expressed in the vessel walls of rejecting allografts (45). However, the consequences of this ex- 


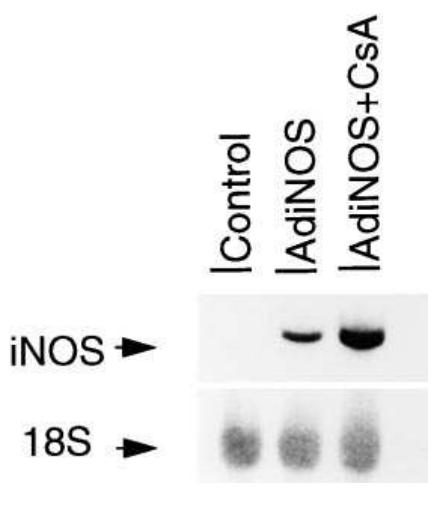

Figure 7. Effect of CsA on AdiNOS. VSMC were transfected with AdiNOS $\left(10^{8} \mathrm{pfu} /\right.$ $\mathrm{ml})$. One group was treated with CsA $\left(10^{-6} \mathrm{M}\right)$. RNA was extracted $24 \mathrm{~h}$ later. CsA had no effect on iNOS mRNA produced by the AdiNOS-transfected VSMC.

pression have not been well-established. Our results indicate clearly that NO produced by iNOS partially suppresses intimal hyperplasia in allograft rejection. The mechanism of this action is not established by our experiments. A direct effect through the suppression of VSMC proliferation is possible. In addition, induced NO could modulate the inflammatory response by inhibiting macrophage accumulation or by exerting cytotoxic effects on infiltrating lymphocytes (24). Other explanations include the inhibition of platelet accumulation and mitogen release, as well as the capacity of NO to protect the endothelium or even promote its regrowth (46).

It is of interest to note that the level of iNOS expressed in the rejecting allograft was inadequate to suppress intimal hyperplasia completely, which may be due to the level of iNOS expression as well as the timing of expression. Others have documented that iNOS expression in aortic allografts does not occur until after the second week. Our results show that provision of additional iNOS early, using an adenoviral-mediated iNOS transfer, suppresses completely allograft arteriosclerosis at $4 \mathrm{wk}$, supporting the concept that endogenous iNOS expression is either insufficient or not present soon enough. This is quite similar to the findings in balloon injury models where iNOS is upregulated in response to the vascular injury but does not suppress completely the development of intimal hyperplasia. Here, too, iNOS gene transfer increases local NO production and in turn inhibits the development of intimal hyperplasia.

CsA is known to accelerate allograft arteriosclerosis, and our data indicate that this is due, at least in part, to the inhibition of iNOS. We and others have shown that CsA inhibits iNOS expression in VSMC stimulated with various cytokines (29-31). This inhibitory effect appears to be due to an inhibition of activation of nuclear factor- $\kappa \mathrm{B}$, a transcription factor required for iNOS transcription. Because the adenovirally expressed human iNOS is driven by the cytomegalovirus promoter, its transcriptional regulation is not subject to CsA suppression, which probably explains why iNOS gene transfer effectively blocked intimal hyperplasia in CsA-treated rat aortic allografts. Where this becomes clinically important is in patients immediately after transplantation or during acute episodes of rejection. These patients characteristically require higher doses of immunosuppressive drugs, including CsA and steroids, both inhibitors of iNOS expression. Thus, patients who are at the greatest risk for the development of graft injury and arteriosclerosis also receive the highest doses of agents that block iNOS expression. The end result is an accelerated development of chronic rejection. With these patients, it is conceivable that AdiNOS gene therapy may allow iNOS expression to continue even in the face of high CsA and steroid doses and may actually suppress the development of this otherwise untreatable disease process.

Adenoviral-mediated gene transfer has been shown to stimulate an inflammatory reaction (47) that might be expected to worsen the rejection process and hasten the eventual development of allograft arteriosclerosis. We saw no difference in intimal hyperplasia between the nontransduced grafts and the grafts transduced with AdlacZ. It is possible that the low adenoviral titers $\left(10^{7} \mathrm{pfu} / \mathrm{ml}\right)$ used here failed to elicit an inflammatory response. It should also be kept in mind that immunosuppressant agents have been shown to suppress adenovirus-mediated inflammation and prolong transgene expression (48), which may be of benefit in any therapeutic application of AdiNOS to prevent allograft arteriosclerosis.

\section{Acknowledgments}

This work was supported by National Institutes of Health grants R29HL-57854 (E. Tzeng), R01-GM-44100 (T.R. Billiar), and R37-AI16869 (R.L. Simmons), as well as a grant from the Thoracic Surgery Foundation for Research and Education (S.M. Pham). Dr. Billiar is the recipient of the George H.A. Clowes, Jr., Memorial Research Career Development Award of the American College of Surgeons. Dr. Pham is the recipient of the American College of Surgeons Faculty Fellowship.

\section{References}

1. Ardehali, A. 1995. Heart transplantation: accelerated graft atherosclerosis. Adv. Card. Surg. 6:195-205.

2. Ventura, H.O., F.W. Smart, D.D. Stapleton, T. Toups, and H.L. Price. 1993. Cardiac allograft vasculopathy: current concepts. J. La. State Med. Soc. 145:195-198.

3. Ventura, H.O., M.R. Mehra, F.W. Smart, and D.D. Stapleton. 1995. Cardiac allograft vasculopathy: current concepts. Am. Heart J. 129:791-799.

4. Kerber, S., A. Rahmel, O. Heinemann-Vechtel, T. Budde, M. Deng, H.H. Scheld, and G. Breithardt. 1995. Angiographic, intravascular ultrasound and functional findings early after orthotopic heart transplantation. Int. J. Cardiol. 49:119-129.

5. Mehra, M.R., H.O. Ventura, F.W. Smart, D.D. Stapleton, T.J. Collins, S.R. Ramee, J.P. Murgo, and C.J. White. 1995. New developments in the diagnosis and management of cardiac allograft vasculopathy. Tex. Heart Inst. J. 22: $138-144$.

6. Gibbons, G.H. 1995. The pathogenesis of graft vascular disease: implications of vascular remodeling. J. Heart Lung Transplant. 14:S149-S158.

7. Mehra, M.R., H.O. Ventura, D.D. Stapleton, and F.W. Smart. 1995. The prognostic significance of intimal proliferation in cardiac allograft vasculopathy: a paradigm shift. J. Heart Lung Transplant. 14:S207-S211.

8. Tanaka, H., S.J. Swanson, G. Sukhova, F.J. Schoen, and P. Libby. 1995. Early proliferation of medial smooth muscle cells in coronary arteries of rabbit cardiac allografts during immunosuppression with cyclosporine A. Transplant. Proc. 27:2062-2065.

9. Russell, P.S., C.M. Chase, H.J. Winn, and R.B. Colvin. 1994. Coronary atherosclerosis in transplanted mouse hearts. I. Time course and immunogenetic and immunopathological considerations. Am. J. Pathol. 144:260-274.

10. Russell, P.S., C.M. Chase, H.J. Winn, and R.B. Colvin. 1994. Coronary atherosclerosis in transplanted mouse hearts. II. Importance of humoral immunity. J. Immunol. 152:5135-5141.

11. Russell, M.E. 1995. Macrophages and transplant arteriosclerosis: known and novel molecules. J. Heart Lung Transplant. 14:S111-S115.

12. Costanzo, M.R. 1995. The role of histoincompatibility in cardiac allograft vasculopathy. J. Heart Lung Transplant. 14:S180-S184.

13. Davis, S.F., A.C. Yeung, I.T. Meredith, F. Charbonneau, P. Ganz, A.P. Selwyn, and T.J. Anderson. 1996. Early endothelial dysfunction predicts the development of transplant coronary artery disease at 1 year posttransplant. Circulation. 93:457-462.

14. Suzuki, J., M. Aikawa, M. Isobe, M. Sekiguchi, Y. Yazaki, and R. Nagai. 1995. Altered expression of smooth muscle and nonmuscle myosin heavy chain isoforms in rejected hearts: a sensitive marker for acute rejection and graft coronary arteriosclerosis. Transplant. Proc. 27:578. 
15. Aziz, S., T.O. McDonald, and H. Gohra. 1995. Transplant arterial vasculopathy: evidence for a dual pattern of endothelial injury and the source of smooth muscle cells in lesions of intimal hyperplasia. J. Heart Lung Transplant. 14:S123-S136.

16. Dusting, G.J., and P.S. Macdonald. 1995. Endogenous nitric oxide in cardiovascular disease and transplantation. Ann. Med. 27:395-406.

17. Tanaka, S., W. Kamiike, T. Ito, S. Nozaki, F. Uchikoshi, M. Miyata, S. Nakata, R. Shirakura, H. Matsuda, and E. Kumura. 1995. Evaluation of nitric oxide during acute rejection after heart transplantation in rats. Transplant. Proc. 27:576-577.

18. Devlin, J., R.M. Palmer, C.E. Gonde, J. O'Grady, N. Heaton, K.C. Tan, J.F. Martin, S. Moncada, and R. Williams. 1994. Nitric oxide generation. A predictive parameter of acute allograft rejection. Transplantation (Baltimore). 58: 592-595.

19. Yang, X., N. Chowdhury, B. Cai, J. Brett, C. Marboe, R.R. Sciacca, R.E. Michler, and P.J. Cannon. 1994. Induction of myocardial nitric oxide synthase by cardiac allograft rejection. J. Clin. Invest. 94:714-721.

20. Russell, M.E., A.F. Wallace, L.R. Wyner, J.B. Newell, and M.J. Karnovsky. 1995. Upregulation and modulation of inducible nitric oxide synthase in rat cardiac allografts with chronic rejection and transplant arteriosclerosis. Circulation. 92:457-464.

21. Radomski, M.W., R.M.J. Palmer, and S. Moncada. 1987. The anti-aggregating properties of vascular endothelium: interactions between prostacyclin and nitric oxide. Br. J. Pharmacol. 92:639-646.

22. Ignarro, L.J., G.M. Buga, K.S. Wood, R.E. Byrns, and G. Chaudhuri. 1987. Endothelium-derived relaxing factor produced and released from artery and vein is nitric oxide. Proc. Natl. Acad. Sci. USA. 84:9265-9269.

23. Worrall, N.K., W.D. Lazenby, T.P. Misko, T.S. Lin, C.P. Rodi, P.T. Manning, R.G. Tilton, J.R. Williamson, and T.B. Ferguson, Jr. 1995. Modulation of in vivo alloreactivity by inhibition of inducible nitric oxide synthase. $J$. Exp. Med. 181:63-70.

24. Kubes, P., M. Suzuki, and D.N. Granger. 1991. Nitric oxide: an endogenous modulator of leukocyte adhesion. Proc. Natl. Acad. Sci. USA. 88:46514655.

25. Langrehr, J.M., R.A. Hoffman, T.R. Billiar, K.K. Lee, W.H. Schraut, and R.L. Simmons. 1991. Nitric oxide synthesis in the in vivo allograft response: a possible regulatory mechanism. Surgery (St. Louis). 110:335-342.

26. von der Leyen, H.E., G.H. Gibbons, R. Morishita, N.P. Lewis, L. Zhang, M. Nakajima, Y. Kaneda, J.P. Cooke, and V.J. Dzau. 1995. Gene therapy inhibiting neointimal vascular lesion: in vivo transfer of endothelial cell nitric oxide synthase gene. Proc. Natl. Acad. Sci. USA. 92:1137-1141.

27. Tzeng, E., L.L. Shears, M.T. Lotze, and T.R. Billiar. 1996. Gene therapy. Curr. Probl. Surg. 33:961-1041.

28. Tzeng, E., L.L. Shears, P.D. Robbins, B.R. Pitt, D.A. Geller, S.C. Watkins, R.L. Simmons, and T.R. Billiar. 1996. Vascular gene transfer of the human inducible nitric oxide synthase: characterization of activity and effects of myointimal hyperplasia. Mol. Med. 2:211-225.

29. Akita, K., G.J. Dusting, and H. Hickey. 1994. Suppression of nitric oxide production by cyclosporine A and FK 506A in rat vascular smooth muscle cell. Clin. Exp. Pharmacol. Physiol. 21:231-233.

30. Marumo, T., T. Nakaki, K. Hishikawa, H. Suzuki, R. Kato, and T. Saruta. 1995. Cyclosporin A inhibits nitric oxide synthase induction in vascular smooth muscle cells. Hypertension (Dallas). 25:764-768.

31. Kunz, D., G. Walker, W. Eberhardt, D. Nitsch, and J. Pfeilschifter. 1995. Interleukin 1 beta-induced expression of nitric oxide synthase in rat renal mesangial cells is suppressed by cyclosporin A. Biochem. Biophys. Res. Commun.
216:438-446.

32. Hunley, T.E., S. Iwasaki, T. Homma, and V. Kon. 1995. Nitric oxide and endothelin in pathophysiological settings. Pediatr. Nephrol. 9:235-244.

33. Hayry, P., A. Mennander, S. Tiisala, J. Halttunen, S. Yilmaz, and T. Paavonen. 1991. Rat aortic allografts: an experimental model for chronic transplant arteriosclerosis. Transplant. Proc. 23:611-612.

34. Moore, W.M., R.K. Webber, G.M. Jerome, F.S. Tjoeng, T.P. Misko, and M.G. Currie. 1994. L- $N^{6}$-(1-iminoethyl)lysine: a selective inhibitor of inducible nitric oxide synthase. J. Med. Chem. 37:3886-3888.

35. Hansson, G.K., Y.J. Geng, J. Holm, P. Hardhammer, A. Wennmalm, and E. Jennische. 1994. Arterial smooth muscle cells express nitric oxide synthase in response to endothelial injury. J. Exp. Med. 180:733-738.

36. Geller, D.A., C.J. Lowenstein, R.A. Shapiro, A.K. Nussler, M. Di Silvio, S.C. Wang, D.K. Nakayama, S.H. Snyder, R.L. Simmons, and T.R. Billiar. 1993 Molecular cloning and expression of inducible nitric oxide synthase from human hepatocytes. Proc. Natl. Acad. Sci. USA. 90:3491-3495.

37. Graham, F.L., J. Smiley, W.C. Russell, and R. Nairn. 1977. Characteristics of a human cell line transformed by DNA from a human adenovirus type 5 . J. Gen. Virol. 36:59-74.

38. Stow, N.D. 1981. Cloning of a DNA fragment from the left-hand terminus of the adenovirus type 2 genome and its use in site-directed mutagenesis. $J$. Virol. 37:171-180.

39. Geller, D.A., A.K. Nussler, M. Di Silvio, C.J. Lowenstein, R.A. Shapiro, S.C. Wang, R.L. Simmons, and T.R. Billiar. 1993. Cytokines, endotoxin, and glucocorticoids regulate the expression of inducible nitric oxide synthase in hepatocytes. Proc. Natl. Acad. Sci. USA. 90:522-526.

40. Marks, D.S., J.A. Vita, J.D. Folts, J.F. Keaney, Jr., G.N. Welch, and J. Loscalzo. 1995. Inhibition of neointimal proliferation in rabbits after vascular injury by a single treatment with a protein adduct of nitric oxide. J. Clin. Invest. 96:2630-2638.

41. Guo, J.P., K.A. Milhoan, R.S. Tuan, and A.M. Lefer. 1994. Beneficial effect of SPM-5185, a cysteine-containing nitric oxide donor, in a rat carotid artery intimal injury. Circ. Res. 75:77-84.

42. Lee, J.S., C. Adrie, H.J. Jacob, J.D. Roberts, Jr., W.M. Zapol, and K.D. Bloch. 1996. Chronic inhalation of nitric oxide inhibits neointimal formation af ter balloon-induced arterial injury. Circ. Res. 78:337-342.

43. Lamas, S., P.A. Marsden, G.K. Li, P. Tempst, and T. Michel. 1992. Endothelial nitric oxide synthase: molecular cloning and characterization of a distinct constitutive enzyme isoform. Proc. Natl. Acad. Sci. USA. 89:6348-6352.

44. Nakayama, D.K., D.A. Geller, M. Di Silvio, G. Bloomgarden, P. Davies, B.R. Pitt, K. Hatakeyama, H. Kagamiyama, R.L. Simmons, and T.R. Billiar. 1994. Tetrahydrobiopterin synthesis and inducible nitric oxide production in pulmonary artery smooth muscle. Am. J. Physiol. 266:L455-L460.

45. Akyurek, L.M., B.C. Fellstrom, Z.Q. Yan, G.K. Hansson, K. Funa, and E. Larsson. 1996. Inducible and endothelial nitric oxide synthase expression during development of transplant arteriosclerosis in rat aortic allografts. Am. J. Pathol. 149:1981-1990.

46. Schmidt, H.H., and U. Walter. 1994. NO at work. Cell. 78:919-925.

47. Newman, K.D., P.F. Dunn, J.W. Owens, A.H. Schulick, R. Virmani, G. Sukhova, P. Libby, and D.A. Dichek. 1995. Adenovirus-mediated gene transfer into normal rabbit arteries results in prolonged vascular cell activation, inflammation, and neointimal hyperplasia. J. Clin. Invest. 96:2955-2965.

48. Jooss, K., Y. Yang, and J.M. Wilson. 1996. Cyclophosphamide diminishes inflammation and prolongs transgene expression following delivery of adenoviral vectors to mouse liver and lung. Hum. Gene Ther. 7:1555-1566. 